\title{
Definition and Overview of Emerging Threats
}

\section{Luther E. Lindler, Eileen Choffnes, and George W. Korch}

\section{INTRODUCTION}

The risks posed by bioterrorism and the proliferation of biological weapons capabilities have increased concern about how the rapid advances in genetic engineering and biotechnology could enable the production of biological weapons with unique and unpredictable characteristics. The nature of the biotechnology problem-indeed the nature of the biological research enterprise-is vastly different from that of theoretical and applied nuclear physics in the late 1930s. Evolving biotechnology presents an inextricably linked combination of opportunity and danger and that distinction turns on projected consequences and attributed intentions at the level of fundamental research. Matthew Meselson gave a stark warning of the potential dangers posed by the destructive applications of biotechnology at the annual meeting of the National Academy of Sciences in May 2000.

"Every major technology-metallurgy, explosives, internal combustion, aviation, electronics, nuclear energy - has been intensively exploited, not only for peaceful purposes but also for hostile ones. Must this also happen with biotechnology, certain to be a dominant technology of the coming century? During the century just begun, as our ability to modify fundamental life processes continues its rapid advance, we will be able not only to devise additional ways to destroy life but will also be able to manipulate it-including the processes of cognition, development, reproduction, and inheritance. A world in which these capabilities are widely employed for hostile purposes would be a world in which the very nature of conflict has radically changed. There in could lie unprecedented opportunities for violence, coercion, repression, or subjugation."

As noted earlier, biotechnology can be used for destructive purposes, but it also holds vast promise for countering bioterrrorism threats. For example, genomic sequence analysis of pathogens can lead to the design of more effective vaccines and antibiotics. Biotechnology may also be used for large-scale production of therapeutics to counter biological agents.

The "dual" nature of biotechnology is evident in recent scientific publications. For example, an Australian research group attempted to sterilize mice by immunizing them against an important reproductive protein (discussed in detail in Subheading 3.3.). To do so, this protein was inserted into the poxvirus genome to produce the antigen in the mice. A gene for interleukin (IL)-4 was also inserted into this poxvirus vector, because 
IL-4 is known to enhance antibody formation. Although the original intent was simply to sterilize the mice, the researchers found that the engineered poxvirus construct was more virulent. The construct resulted in the death of genetically resistant, vaccineprotected, and wildtype mice. This example illustrates the current potential, through biotechnology, to enhance the potency of disease-causing agents.

Because the fundamental knowledge from which these dangers emerge is commonplace around the world and the potential benefits of biotechnology for medicine and defense are too great, efforts to prohibit or reverse such research and investigations may be futile, at best. However, the potential adverse impacts associated with the exploitation of these technological advances over the next 5-15 yr cannot be ignored. History has demonstrated that research in biology conducted without any military application in mind may still contribute to the production of biological weapons. In this context, the next generation of biological warfare (BW) agents may mimic physiologically active compounds and disregulate fundamental life processes including reproduction, apoptosis, cognition, and enhancement or suppression of the immune response. Microbes used for bioremediation purposes may also be modified to target strategic materials and infrastructures.

Dr. Anna Johnson-Winegar, Deputy Assistant to the Secretary of Defense for Chemical and Biological Defense has made the following remarks.

\begin{abstract}
"[Although] gene-based weaponry is not currently a credible threat, the sophisticated methods which we now have to probe the human genome, . ., introduce the possibility that these techniques may be used to create weapons. Viruses may be developed that can be genetically targeted to a particular nationality or race based on certain genetic characteristics. Biological weapons may be created that are tailored to produce symptoms weeks after exposure but are contagious much earlier, allowing the agent to spread for extended periods prior to medical experts becoming aware that an epidemic has started. Biological agents may be used to produce debilitating but not fatal diseases, requiring the use of tremendous resources for palliative care. The strain on the medical resources and psychological strength of a society could potentially be crippling."
\end{abstract}

The current military Biodefense Research Program (BDRP) focuses on agents listed on the Department of Defense (DOD) validated threat list. With the birth of recombinant DNA technology in the early 1970s, the possibility that new agents might be constructed for offensive purposes became a reality. Military planners in the BDRP program office began to seriously consider this possibility in the early to middle 1990s. Their concerns were borne out with the defection of one of the leaders in the former Soviet Union offensive bioweapons programs. Ken Alibeck described a significant project within this program specifically aimed at the use of recombinant technologies to modify classic agents as well as develop new agents with unique pathogenic properties. This program was referred to as "bonfire" in his book about covert former Soviet Union bioweapons development (3). The bonfire project attempted to make relatively simple (by today's standards) modifications of agents such as the incorporation of antibiotic resistance genes in classic bacterial agents. Russian bioweaponeers were also purported to have modified classic agents such as Yersinia pestis such that they produced dramatically different pathologies compared with the unaltered pathogen. Interestingly, some hints of these activities were published in the open literature although many have not been independently confirmed. 
The weapons alluded to in the previous paragraph all began as one of the classic agents considered for use in BW. However, the possibility exists for development of chimeras that do not exist in nature through the use of recombinant technologies. The study and development of agents isolated from nature that are yet to be discovered is also a possibility that must be considered in planning for future threats. Possible examples of both of these potential agents will be discussed later. However, it should be pointed out that the intent of this chapter is not to specifically discuss the weaponization of these agents but more to address the fact that these bioweapons are a distinct set of unconventional agents that require unconventional countermeasures to deter their possible development and use.

From what was aforementioned, it is clear that emerging threats can be divided into two groups. The first are ones that began with a classic platform or agent, this is the weaponization of disease agents. The second group would be comprised of agents that do not exist in nature and are produced by man. It should be pointed out that the definitions of these two groups may, in some cases, be arbitrary because classic agents may produce new or mixed pathologies after modification. In this instance, the question is when does the agent cease to be the classic agent such that it should be considered a new agent? The purpose of this chapter is to introduce the reader to these current and future possibilities as well as give examples of modified agents that appear in the open literature.

\subsection{Definition of a Validated Threat That Has Been Modified}

Agents in this category are ones that fall into the classic agents but have been modified to circumvent normal countermeasures or therapies. Some in the community refer to these as "improved agents." Examples of these will be discussed in Subheading 2. and include agents made antibiotic resistant or vaccine resistant.

\subsection{Definition of Potential Threats That Could Be Developed}

These agents are ones that may include a modified classic agent that now produces new and unique pathology. Modified agents could be created by recombinant technologies. These agents include generation after next organisms or toxins that are manmade to target specific biological systems. Agents in this category might also include ones that have newly emerged and are amenable to weaponization or that encode some properties necessary for pathogenesis but lack others that are critical to cause disease. These may be referred to as "advanced" agents by some in the community. Possible examples of these are presented in Subheading 3. It is important to note that these agents can be created outside natural evolutionary pressures.

\section{EXAMPLES OF MODIFIED VALIDATED THREATS}

\subsection{Recombinant Anthrax}

In 1997, the DOD medical community was alerted to just how serious a threat genetic modification was to their planned anthrax vaccination program for military personnel. A small group of Russian scientists at the State Research Center for Applied Microbiology in Oblensk published a paper in the journal Vaccine, which demonstrated that the addition of a single gene to a strain of Bacillus anthracis could cause disease in vaccinated animals (21). This research center was one that Alibeck had mentioned in 
his book as specializing in constructing recombinant threat agents (3). These researchers added a cereolysin gene from Bacillus cereus to a fully virulent $B$. anthracis strain. The cereolysin $A B$ genes encode a functional hemolysin. Hamsters immunized with live $B$. anthracis harboring only pOX1 encoding the anthrax toxin and not pOX2 encoding the capsule displayed an immune index of 140 when challenged with the unmodified (cereolysin negative) recombinant. In contrast, hamsters immunized with the same strain and challenged with the recombinant cereolysin-producing strain were not protected (immune index approx 1). Thus, mice could be well-protected by live immunization with a crippled strain of $B$. anthracis but were not protected when challenged with the cereolysin producing recombinant organism. This "vaccine breakthrough" could be overcome if the cereolysin gene was included in the live $B$. anthracis vaccine strain used to immunize the animals. These results brought into question the utility of vaccination of the entire military population and weather simple genetic modification might circumvent that program. It should be noted that these results have not been independently confirmed at this time.

\subsection{Antibiotic Resistance}

The classic bacterial biothreat agents are generally susceptible to most antibiotics used to treat the infections $(5,9,11,24,26)$. However, multidrug resistance has been reported for $Y$. pestis but is confined to the island of Madagascar $(13,15)$. Doxycycline is one of the primary antibiotics recommended for treatment of infections caused most of the high-risk bacterial threat agents (US Army Medical Research Institute if Infectious Diseases Medical Management of Biological Casualties Handbook, http:// www.usamriid.army.mil/education/bluebook.html). The Russian offensive program is purported to have invested in the generation of recombinant organisms that were resistant to the common therapies such as antibiotic treatment (3). This is consistent with the desire to produce offensive biological weapons for which there was no current cure (3). The idea that they were producing antibiotic-resistant BW agents is supported by several publications in the open literature. Gorelov et al. generated a multidrug resistant vaccine strain of Brucella abortus (14). This strain was resistant to doxycycline, streptomycin, ampicillin, and rifampicin. The drug-resistant strain was generated by spontaneous selection for rifampicin resistance and by transformation with a "hybrid" plasmid. The researchers went on to demonstrate that the vaccine strain retained the ability to immunize animals against brucellosis. This latter point was important because recombinant strains of pathogenic organisms can be altered in terms of biological properties in some instances. In relation to recombinant antibiotic resistant $B$. anthracis, Pomerantsev and Staritsyn (22) studied the stability of a tetracycline resistance plasmid pCET in vegetative cells and in spores. These studies included conditions for stable maintenance, complete loss and integration of the plasmid into the chromosome of $B$. anthracis. Experiments to test stability of the added phenotype would be necessary for the development of antibiotic resistant $B$. anthracis strains as a BW agent, which is consistent with Alibeck's assertion that the former Soviet Union offensive BW program developed strains of the organism that were resistant to five different antibiotics (3). Generation of multiply antibiotic-resistant strains of Pseudomonas mallei (Burkholderia mallei) and P. pseudomallei (B. pseudomallei), which cause glanders and melioidosis, respectively, have also been described in the open literature $(1,2,25)$. 
These papers describe genetic manipulation by transformation and conjugation as well as the effect of plasmid maintenance has on virulence of the organism. Taken together, these papers as well as others described in Subheading 2. of this chapter, demonstrate the emphasis that the former Soviet Union BW offensive program placed on alteration of antibiotic resistance of the agents under development.

\subsection{Recombinant Francisella tularensis}

In 1993, Borzenkov et al. published experiments describing the construction of an attenuated strain of Francisella tularensis harboring a construct expressing the human $\beta$-endorphin gene (6). The production of the recombinant bioactive peptide was reported to cause dose-dependent effects consistent with endorphin expression in mice infected with the recombinant strain. This is the first report of a bioactive peptide being delivered by a threat agent and having a physiological effect in animals. The following year, the same group reported construction of recombinant strains of Yersinia, Francisella, and Brucella producing human endorphin (7). Although not specifically reported for obvious reasons, these recombinant organisms might be expected to produce altered pathology following infection that might confound treatment of the disease. For example, the animal might demonstrate signs of the bacterial infection but also signs of an overdose of a drug such as an opiate. Although these effects are theoretically possible, it is unknown what real effects recombinant organisms of this type might have.

\section{POTENTIAL THREATS}

\subsection{Organisms That Develop in Nature That Might Be Weaponized}

Many traits must be considered when BW agents are developed. These concepts are briefly discussed in Chapter 3. Accordingly, it is conceivable that a pathogen could emerge in the future through natural selection that meets the criteria for weaponization, and therefore, might become a threat agent. There have been numerous new diseases in recent history any of which could potentially be characterized for weaponization. The most recent example of a newly emerged disease was the Sudden Acute Respiratory Syndrome (SARS) epidemic (27). The virus that causes SARS might be considered as a BW agent because it was highly infectious, communicable and caused mortality. Besides being an example of a natural emerging threat, SARS is also an example of how we might recognize, respond, and limit damage because of a bioterrorist attack. Accordingly, planners for future bioterrorist attacks could use the public health and research community response to the SARS epidemic to gain insight into how to possibly reduce the impact of agent release.

\subsection{Low Virulence or Nonpathogenic Organisms That Could Be Genetically Modified}

A more theoretical group of organisms that might be developed into BW agents in the laboratory are ones that are of low pathogenicity but that encode some factors associated with virulence. The addition of specific genes could then result in the creation of a more virulent organism. This process can be thought of as the laboratory equivalent of what occurs in nature. The process from nonpathogen to pathogen is believed to be primarily driven by lateral gene transfer events $(20)$. The resulting pathogen depends 
on the traits that are added to the ancestral bacteria. The study of evolution of pathogens in this manner has been greatly supported and accelerated by genomic sequencing efforts and comparisons between completed genomes. One specific example of the close relationship between nonpathogenic and highly pathogenic members of a genus can be see in the comparison of $Y$. pestis and Yersinia pseudotuberculosis. These two organisms are highly related at the whole genome level yet cause vastly different diseases (8). A detailed description of the differences between $Y$. pestis and Y. pseudotuberculosis can be found in Chapter 22. The emergence of a new pathogenic organism is thought to be through "genetic sampling" such that new combinations of genetic materials coalesce and allow an organism to occupy a new niche in nature. Theoretically, this might be possible to duplicate in the laboratory using recombinant techniques and, therefore, must be considered in future preparations to counter bioterrorism and BW threats.

\subsection{The Mousepox That Roared}

A relatively recent publication by Jackson et al. (16) had important implications in the field of genetically engineered or emerging threats. These researchers demonstrated that a modified mousepox virus, ectromelia (ECTV) could: a) cause lethal infections in normally genetically resistant mice; and b) cause lethal infections in mice previously immunized against infection with ECTV. The genetic modification included in this strain of ECTV was the inclusion of the gene encoding the normal cytokine IL-4 into the genome of the virus. These experiments were performed under the auspices of enhancing the immune response to other recombinant antigens carried on the virus. The creation of the supermousepox and the possibility that similar experiments could be done with smallpox had obvious implications on further development of an already formidable bioweapon. These implications were noted in prominent scientific journals and reignited discussions about the open nature of publication of scientific results and implications such publications have for national security $(4,12,19)$.

\section{COUNTERMEASURES: WHAT CAN WE DO TO PREPARE?}

\subsection{Genome Sequencing and Database Development}

Possibly one of the greatest difficulties will be the identification of the new agent that is causing the disease. This was seen more than $20 \mathrm{yr}$ ago when the agent of Legionnaire's disease could not be isolated. Of course the first step is the isolation of the agent but then soon follows the identification needed to help provide proper therapy for infected patients. The recent emergence of SARS is a good example of how rapid sequencing capability can provide useful information during an infectious disease epidemic. Two different groups were able to grow and isolate the virus from infected tissue culture cells at about the same time $(10,17)$. These studies used electron microscopic examination, random polymerase chain reaction sequencing, and immunoreactivity to identify the agent as a coronavirus. Two weeks later, the complete genome sequence of the SARS virus was reported by two independent groups (18,23). Knowledge of the complete genome sequence allowed the exact identification of the virus as well as the determination of the phylogeny that gave rise to this new strain. The fact that SARS has a relatively small genome of approx $27 \mathrm{~kb}$ facilitated the rapid sequencing, assembly, and molecular characterization of this newly emerged virus. Of course, 
this would be more difficult with larger bacterial genomes that are megabases in size. However, complete sequence information may not be necessary to determine the identity of the organism involved in the outbreak at least sufficiently enough to allow proper treatment.

The databases used to analyze the genome sequences obtained in the earlier SARS example were standard databases such as GenBank. Development of more sophisticated databases that concentrate on virulence factors, pathogen genetic traits, and other characteristics associated with pathogenicity may be needed. Well-annotated and developed datasets of this type will be useful for research purposes such as genomic sequencing projects and the identification of potential virulence properties. Genome sequencing projects and use of databases of this type may be useful in the identification of low-virulence or nonpathogenic organisms that might be genetically modified (see Subheading 3.2.). A virulence factor database of this type would also be useful for the characterization of novel agents postevent as well.

\subsection{Genotyping}

The development of a large genotyping database for the primary threat agents would allow us to identify newly emerging strains of these organisms. The success of such a project would depend greatly on international cooperation because most of the diseases caused by the primary agents are not prevalent in the United States. Not only would such a genetic fingerprint database help identify new strains of these organisms, but it would also support attribution efforts in the event of a bioterrorist attack. Accordingly, a genotype database capability is absolutely critical for monitoring and countering emerging threats. More information can be found about genotyping BW pathogens in Chapter 21.

\subsection{Detailed Study of Emerged Pathogens}

The investigation of how new pathogens arise in nature could lend insight into how an aggressor might modify an organism to use as a new threat. The more details that are learned about pathogenesis of various infectious diseases will greatly enhance our ability to identify and respond to the use of a novel agent. Primarily, the more we know about the "pathosphere" will enhance our ability to detect traits of pathogens and characterize these new organisms. Investment in monitoring organisms circulating in animals will also support our ability to avoid "tactical surprise" because many diseases such as SARS and influenza arise in the human population through close contact with domestic animals. In fact, emerging infectious disease surveillance is a natural partner in the fight against bioterrorism. Many programs within this discipline have been harnessed for this purpose (see Chapter 17 for more details).

\subsection{Investment in Research on Nonspecific Immunity}

In the event that a genetically modified agent were to be used, it is likely that we would not have on-hand a specific vaccine or therapeutic. Therefore, it is likely that we will be forced to rely on nontraditional measures for treatment of infected individuals. These treatments could come in the form of cytosine and guanosine (see Chapter 14) or the use of specific immunomodulators. Research in this area could be critical for countering an attack with a previously unknown threat agent. 


\section{CONCLUSIONS}

The number of scenarios that an ill-intended group might invent is almost limitless certainly to the point that all possibilities could not be programmed in any reasonable manner. Even with good intelligence, we cannot be assured that a covert group will not develop novel agents. For this reason, our best defense is one of developing knowledge in key areas that will be useful should an event occur. This chapter attempts to address some of these key areas including genomic sequencing, informatics, forensic genotyping, emerging disease monitoring, and research. Linking all of these elements together to form a national shield against the use of modified or novel agents is our best and only viable defense.

\section{ACKNOWLEDGMENTS}

We thank Ted Plasse for reviewing the manuscript and making helpful suggestions.

\section{DISCLAIMER}

This chapter is the opinion of the authors and does not represent the view of the Department of Defense or of the Department of the Army.

\section{REFERENCES}

1. Abaev, I. V., Akimova, L. A., Shitov, V. T., Volozhantsev, N. V., and Svetoch, E. A. (1992) Transformation of pathogenic pseudomonas by plasmid DNA. Mol. Gen. Mikrobiol. Virusol. 17-20.

2. Abaev, I. V., Astashkin, E. I., Pachkunov, D. M., Stagis, N. I., Shitov, V. T., and Svetoch, E. A. (1995) Pseudomonas mallei and Pseudomonas pseudomallei: introduction and maintenance of natural and recombinant plasmid replicons. Mol. Gen. Mikrobiol. Virusol. 28-36.

3. Alibeck, K. and Handleman, S. (1999) Biohazard. Random House, New York.

4. Atlas, R. M. (2002) Public health. National security and the biological research community. Science 298, 753, 754.

5. Bodur, H., Balaban, N., Aksaray, S., Yetener, V., Akinci, E., Colpan, A., and Erbay, A. (2003) Biotypes and antimicrobial susceptibilities of Brucella isolates. Scand. J. Infect. Dis. 35, 337, 338.

6. Borzenkov, V. M., Pomerantsev, A. P., and Ashmarin, I. P. (1993) The additive synthesis of a regulatory peptide in vivo: the administration of a vaccinal Francisella tularensis strain that produces beta-endorphin. Biull. Eksp. Biol. Med. 116, 151-153.

7. Borzenkov, V. M., Pomerantsev, A. P., Pomerantseva, O. M., and Ashmarin, I. P. (1994) Study of nonpathogenic strains of Francisella, Brucella and Yersinia as producers of recombinant beta-endorphin. Biull. Eksp. Biol. Med. 117, 612-615.

8. Brubaker, R. R. (1991) Factors promoting acute and chronic diseases caused by yersiniae. Clin. Microbiol. Rev. 4, 309-324.

9. Coker, P. R., Smith, K. L., and Hugh-Jones, M. E. (2002) Antimicrobial susceptibilities of diverse Bacillus anthracis isolates. Antimicrob. Agents Chemother. 46, 3843-3845.

10. Drosten, C., Gunther, S., Preiser, W., et al. (2003) Identification of a novel coronavirus in patients with severe acute respiratory syndrome. N. Engl. J. Med. 348, 1967-1976.

11. Esel, D., Doganay, M., and Sumerkan, B. (2003) Antimicrobial susceptibilities of 40 isolates of Bacillus anthracis isolated in Turkey. Int. J. Antimicrob. Agents 22, 70-72.

12. Finkel, E. (2001) Australia. Engineered mouse virus spurs bioweapon fears. Science 291, 585.

13. Galimand, M., Guiyoule, A., Gerbaud, G., et al. (1997) Multidrug resistance in Yersinia pestis mediated by a transferable plasmid. N. Engl. J. Med. 337, 677-680. 
14. Gorelov, V. N., Gubina, E. A., Grekova, N. A., and Skavronskaia, A. G. (1991) The possibility of creating a vaccinal strain of Brucella abortus 19-BA with multiple antibiotic resistance. Zh. Mikrobiol. Epidemiol. Immunobiol. 2-4.

15. Guiyoule, A., Gerbaud, G., Buchrieser, C., et al. (2001) Transferable plasmid-mediated resistance to streptomycin in a clinical isolate of Yersinia pestis. Emerg. Infect. Dis. 7, 43-48.

16. Jackson, R. J., Ramsay, A. J., Christensen, C. D., Beaton, S., Hall, D. F., and Ramshaw, I. A. (2001) Expression of mouse interleukin-4 by a recombinant ectromelia virus suppresses cytolytic lymphocyte responses and overcomes genetic resistance to mousepox. J. Virol. 75, 1205-1210.

17. Ksiazek, T. G., Erdman, D., Goldsmith, C. S., et al. (2003) A novel coronavirus associated with severe acute respiratory syndrome. N. Engl. J. Med. 348, 1953-1966.

18. Marra, M. A., Jones, S. J., Astell, C. R., et al. (2003) The Genome sequence of the SARSassociated coronavirus. Science 300, 1399-1404.

19. Mullbacher, A. and Lobigs, M. (2001) Creation of killer poxvirus could have been predicted. J. Virol. 75, 8353-8355.

20. Ochman, H., Lawrance, J. G., and Groisman, E. A. (2000) Lateral gene transfer and the nature of bacterial innovation. Nature 405, 299-304.

21. Pomerantsev, A. P., Staritsin, N. A., Mockov Yu, V., and Marinin, L. I. (1997) Expression of cereolysine $\mathrm{AB}$ genes in Bacillus anthracis vaccine strain ensures protection against experimental hemolytic anthrax infection. Vaccine 15, 1846-1850.

22. Pomerantsev, A. P. and Staritsyn, N. A. (1996) Behavior of heterologous recombinant plasmid pCET in cells of Bacillus anthracis. Genetika 32, 500-509.

23. Rota, P. A., Oberste, M. S., Monroe, S. S., et al. (2003) Characterization of a novel coronavirus associated with severe acute respiratory syndrome. Science 300, 1394-1399.

24. Smith, M. D., Vinh, D. X., Nguyen, T. T., Wain, J., Thung, D., and White, N. J. (1995) In vitro antimicrobial susceptibilities of strains of Yersinia pestis. Antimicrob. Agents Chemother. 39, $2153,2154$.

25. Verevkin, V. V., Volozhantsev, N. V., Miakinina, V. P., and Svetoch, E. A. (1997) Effect of TRA-system of plasmids RP4 and R68.45 on Pseudomonas mallei virulence. Vestn. Ross. Akad. Med. Nauk. 37-40.

26. Wong, J. D., Barash, J. R., Sandfort, R. F., and Janda, J. M. (2000) Susceptibilities of Yersinia pestis strains to 12 antimicrobial agents. Antimicrob. Agents Chemother. 44, 1995, 1996.

27. Ziebuhr, J. (2003) SARS-unprecedented global response to a newly emerging disease. Int. J. Med. Microbiol. 293, 229-231. 